diagnosis. Patients with a diagnosis of irritable bowel syndrome or a record of opioid use were excluded. Records were analysed from 1 January 2008 to 31 December 2011 for the 12-month period following the first constipation diagnosis. Mortality, frequency of comorbidities, number of healthcare consultations, and total laxative prescriptions and costs were recorded and stratified by sex and age group (age 18-49, 50-64, 65-74, and $\geq 75$ years). For patients with less than 12 months follow-up, data on costs, healthcare contacts and prescriptions were adjusted to an annual rate using a simple linear projection. Regression analyses were performed on a case basis to estimate the impact of covariates on laxative costs.

Results Overall, 10371 patients with chronic constipation were identified, of whom $63 \%$ were women and $53 \%$ were $\geq 75$ years old. Mortality during the 12 -month follow up period was $8.4 \%$. The most common co-morbidities were primary hypertension (32\%) and type 2 diabetes mellitus (13\%). Patients had a mean of 27.7 consultations (standard deviation [SD] 24.0), including 4.0 (SD 9.8) for constipation-related care, in the 12-month follow up period. In total, $92 \%$ of patients were prescribed laxatives; patients had on average 8.3 (SD 14.0) laxative prescriptions in the 12 month follow up, which using an average prescription cost of $£ 4.28$ resulted in a mean cost to the National Health Service of $£ 35.41 /$ person/year in 2011 GBP. An increase in prescription laxative cost was significantly associated with increasing age group, the presence of Parkinson's disease (proportional cost increase [PCI] $1.29, \mathrm{p}<0.0005)$, type 2 diabetes mellitus (PCI 1.08, $\mathrm{p}<0.025$ ) and multiple sclerosis (PCI 1.51, $\mathrm{p}<0.0005$ ).

Conclusion In this analysis of UK electronic medical records using the IMS UK Disease Analyzer database, the majority of patients with chronic constipation were elderly, and over $90 \%$ were prescribed laxatives. Higher treatment costs were associated with increased age and co-morbidity.

Disclosure of Interest M. Radford Employee of: IMS Health, which received funding from Shire to carry out this research., E. Bloomfield Employee of: IMS Health, which received funding from Shire to carry out this research., A. Joseph Employee of: Shire.

\section{PWE-179 A SURVEY ON PATIENT WELLBEING AND PATIENT COMFORT DURING OESOPHAGEAL INTUBATIONS. A MULTICENTRE STUDY}

${ }^{1} \mathrm{M}$ Brennan*, ${ }^{*} \mathrm{~T}$ Moran, ${ }^{1} \mathrm{P}$ Lawlor, ${ }^{2} \mathrm{~L}$ Barry, ${ }^{3} \mathrm{M}$ Treacy, ${ }^{4} \mathrm{JV}$ Reynolds, ${ }^{4} \mathrm{R}$ Narayanasamy. ${ }^{1}$ Gl Function Unit, St James Hospital, Dublin 8, Ireland; ${ }^{2}$ Clinical Measurement Lab, Mercy University Hospital, Cork, Ireland; ' University College Hospital, Galway, Co. Galway, Ireland; ${ }^{4}$ Department of Surgery, St James Hospital, Dublin 8, Ireland

10.1136/gutjnl-2014-307263.439
Introduction A Multicentre survey was undertaken from November 2013 until January 2014 on 80 patients (45f Vs $35 \mathrm{~m}$ ) undergoing oesophageal manometry and/or oesophageal $24 \mathrm{hr} \mathrm{pH} /$ impedance study. The aim of this study was to determine the overall wellbeing of the patient during their investigation. Oesophageal intubations are invasive techniques which many patients find quite distressing. These studies require the patient to be alert and to be able to swallow liquid boluses. In order to retain their normal oesophageal function, sedation or oral anaesthetic spray is not routinely administered. This study was compiled to obtain specific patient data from Gastrointestinal (GI) Units throughout Ireland by conducting a survey post patient procedure.

Methods A survey containing short answer questions was devised and GI units providing a service in GI Physiology testing were asked to participate. The patient was asked to answer either 'Yes' or 'No' to the questions or score the answers to the questions with a value from $0-10$ with 10 being the most severe scale of discomfort/anxiety and 0 being the least.

Results Table 1 below shows the results obtained from the multicentre survey.

The reasons given for failed intubations included patient anxiety, nasal sensitivity and previous traumatic Endoscopy experience. With regards to patient anxiety prior to investigation; no option for general anaesthetic, sedation, nasal spray, throat spray, and the fear of the unknown were the main reasons for the high patient anxiety scores.

Conclusion Appropriately trained GI Physiologists achieved a 96.3\% rate of successful oesophageal intubations. Despite this, patient anxiety in anticipation of their procedure is relatively high. With the option of a nasal spray, this survey suggests that patient anxiety levels prior to their investigation would be reduced, thus making the intubation a more pleasant, tolerable and less traumatic experience.

Disclosure of Interest None Declared.

\section{PWE-180 A SURVEY EVALUATING GENERAL PRACTITIONERS', GASTROENTEROLOGISTS' AND EXPERTS' DIAGNOSTIC APPROACHES TO INFLAMMATORY BOWEL DISEASE, IRRITABLE BOWEL SYNDROME AND CHRONIC CONSTIPATION IN FIVE EUROPEAN COUNTRIES}

${ }^{1} V$ Andresen, ${ }^{2} \mathrm{P}$ Whorwell*, ${ }^{3} \mathrm{~J}$ Fortea, ${ }^{4} \mathrm{~J}$ Milce. ${ }^{1} /$ sraelitisches Krankenhaus, Hamburg, Germany; ${ }^{2}$ Wythenshawe Hospital, Manchester, UK; ${ }^{3}$ Almirall S. A., Barcelona, Spain; ${ }^{4}$ Kantar Health, Paris, France

10.1136/gutjnl-2014-307263.440

\begin{tabular}{|c|c|c|c|}
\hline & Total & Female & Male \\
\hline Mean Age (years) & 49.23 & 53.58 & 43.63 \\
\hline Successful intubation rate\% & $96.3 \%$ & $95.6 \%$ & $97.1 \%$ \\
\hline Successful intubation rate in patients & $94.4 \%$ & $94.4 \%$ & $94.4 \%$ \\
\hline \multicolumn{4}{|l|}{$>40$ years } \\
\hline Successful intubation rate in patients & $100 \%$ & $100 \%$ & $100 \%$ \\
\hline \multicolumn{4}{|l|}{$<40$ years } \\
\hline Mean anxiety score prior to investigation * & 4.73 & 5.47 & 3.77 \\
\hline Mean anxiety score post procedure * & 1.96 & 2.26 & 1.59 \\
\hline Mean discomfort level & 4.91 & 4.93 & 4.89 \\
\hline Number of patients who would be anxious if studies had to be repeated & 32.55 & $35.56 \%$ & $28.57 \%$ \\
\hline Number of patients who would choose to have an anaesthetic spray administered & $61.25 \%$ & $66.67 \%$ & $54.29 \%$ \\
\hline
\end{tabular}


Abstract PWE-180 Table 1 Ability to make a diagnosis for each patient vignette

\begin{tabular}{llll}
\hline & IBS-C & IBS-D & IBD \\
\hline Type of physician & Experts / GEs / GPs & Experts / GEs / GPs & Experts / GEs / GPs \\
Correct diagnosis & $88 \% / 56 \% / 31 \%$ & $92 \% / 72 \% / 64 \%$ & $92 \% / 87 \% / 85 \%$ \\
Incorrect diagnosis & $4 \% / 4 \% / 5 \%$ & $8 \% / 12 \% / 14 \%$ & $4 \% / 13 \% / 14 \%$ \\
Don't know & $8 \% / 40 \% / 64 \%$ & $-/ 16 \% / 22 \%$ & $4 \% /-/ 1 \%$ \\
\hline
\end{tabular}

Introduction The confident diagnosis of chronic abdominal conditions can be challenging. This study assessed the diagnostic process in irritable bowel syndrome with constipation (IBSC), irritable bowel syndrome with diarrhoea (IBSD), inflammatory bowel disease (IBD) and chronic constipation (CC).

Methods Online interviews were conducted with 25 experts, 100 gastroenterologists (GEs) and 104 general practitioners (GPs) from Germany, Spain, France, Italy and the United Kingdom to explore perception, attitude and diagnostic approach to IBS. Physicians were also presented patient vignettes describing a typical case of IBS-C, IBS-D, IBD and CC, respectively. For each vignette, participants were asked to make a diagnosis and to give details on further clinical investigations and management of each case.

Results The CC and IBS-C vignettes caused most diagnostic difficulties. For the IBS-C vignette, most GEs and GPs who did not make a correct diagnosis were unsure of the diagnosis. In contrast, most physicians who did not make a correct diagnosis for the CC vignette gave an incorrect diagnosis of IBS-C.

Physicians' confidence level in their diagnosis was 7.0/9 for the IBS-D vignette, 6.8/9 for IBS-C and 6.7/9 for CC. The score was lowest for IBD (6.3) as most physicians stated they would wait for the results of further investigations prior to diagnosis. Experts were most likely to endorse a positive approach to the diagnosis of IBS, IBD or CC, whereas GEs and GPs preferred to adopt a diagnosis by exclusion approach. For the IBS and CC vignettes, most physicians' next action would be to prescribe treatment. However, for the IBD vignette, the next action would be to conduct tests. Most experts (96\%) and GEs (73\%) claimed to have a good knowledge of the Rome III diagnostic criteria, compared to only $15 \%$ of GPs.

Conclusion The study highlights some of the difficulties experienced by GPs, GEs and experts in diagnosing chronic abdominal conditions. Physicians found differentiating between IBS-C and $\mathrm{CC}$ to be particularly challenging. Diagnostic criteria designed for research purposes may not necessarily be applicable in standard clinical practice.

Study funded by Almirall S. A.

Disclosure of Interest V. Andresen Consultant for: Almirall, Astra Zeneca, Norgine, Shire, Conflict with: Almirall, Abbvie, Aptalis, Ardeypharm, Norgine, Shire, Mundipharma, Falk, P. Whorwell: None Declared, J. Fortea Employee of: Almirall S. A., J. Milce Grant/research support from: Almirall, Consultant for: Kantar Health.

\section{PWE-181 QUANTITATIVE ASSESSMENT OF GLOBAL SMALL BOWEL MOTILITY IN CHRONIC INTESTINAL PSEUDO-OBSTRUCTION AND CONTROLS: A PRELIMINARY STUDY}

${ }^{1}$ SK Butt*, ${ }^{2} \mathrm{~A}$ Menys, ${ }^{2} \mathrm{D}$ Atkinson, ${ }^{2} \mathrm{~A}$ Plumb, ${ }^{2} \mathrm{~S}$ Taylor, ${ }^{1} \mathrm{~N}$ Zarate-Lopez, ${ }^{1} \mathrm{~A}$ Emmanuel. ${ }^{1}$ Gastroenterology, UCL London, UK; ${ }^{2}$ Centre for Medical Imaging, UCL, London, UK
Introduction In this preliminary report, we present the initial results of a prospective investigation comparing MRI quantified global small bowel motility in healthy controls and patients with proven clinical and radiological Chronic Intestinal pseudo-obstruction (CIPO). Diagnosis is initially difficult and often delayed, many patients undergoing unnecessary surgical intervention prior to final diagnosis. MRI offers a potential non-invasive modality of diagnosis and monitoring, employing post-processing quantitation of global metrics describing small bowel motility ${ }^{1}$.

Methods Subject selection: 11 healthy non-smoking volunteers (7 Male, mean age 33[22 to 48]) and 5 CIPO patients (3 Male, mean age $53[32$ to 82$]$ ) were recruited. CIPO patients stopped any medications that influenced small bowel motility for one week prior to scan including opioids, anti-emetics and anti-diarrhoeals. Study overview: Participants underwent a single MRI motility scan before and immediately after an injection of $0.5 \mathrm{mg}$ IV neostigmine, a cholinomimetic with potent prokinetic action. Statistics: Data normality was assessed using Shapiro-Wilk testing. 1) Baseline motility was compared in CIPO patients and controls. 2) Percent change in motility between baseline and post-neostigmine was compared between groups. Difference in means were tested using Welch's T-test.

Results

1. Mean baseline small bowel motility scores in CIPO patients was 0.19AU (range 0.1 to 0.25 ) and in controls 0.35AU (range 0.275 to 0.37 ) with a statistically significant difference of $0.17 \mathrm{AU}, \mathrm{P}=0.0026$ (CI 0.09 to 0.23 ).

2. The mean percent increase in small bowel motility scores in CIPO patients following noestigmine was 29\% (95\% CI from 19 to $50 \%$ ) and in controls $10 \%$ (range 0 to 34 ) with a statistically significant difference in groups response to neostigmine of $19 \%, \mathrm{P}=0.029$ (95\% CI from 4 to $40 \%$ ).

Conclusion This study demonstrated significant differences in both resting and cholinomimetic-induced global motility between CIPO patients and healthy controls. Despite marked bowel distension in the CIPO patients, motility appeared present but reduced compared to controls, and responded to provocation with neostigmine suggesting the bowel still exhibits the expected pro-kinetic effects following pharmacological stimulation. With just five patients this is a preliminary study, nevertheless initial results appear promising and support our ongoing investigation program.

\section{REFERENCE}

1 Menys et al. Global Assessment Radiol 2013;269(2):443-50

2 Odille et al. Qu, UKantitative assessment MRM 2012:68(3):783-93

Disclosure of Interest None Declared.

\section{PWE-182 DYSMOTILITY IN PARKINSON'S DISEASE CORRELATES TO GUT SYMPTOMS: FINDINGS OF A WIRELESS MOTILITY CAPSULE STUDY}

${ }^{1} \mathrm{SK}$ Butt ${ }^{*},{ }^{1} \mathrm{R}$ Leung, ${ }^{2} \mathrm{~A}$ Bala, ${ }^{2} \mathrm{~K}$ Bhatia, ${ }^{1} \mathrm{~A}$ Raeburn, ${ }^{1} \mathrm{~A}$ Emmanuel. ${ }^{1}$ Gastroenterology, UCL London, UK; ${ }^{2}$ Neurology, National Hospital for Neurology and Neurosurgery, London, UK

10.1136/gutjnl-2014-307263.442 